\title{
Common-mode suppressed differential bandpass filter based on open complementary split ring resonators (OCSRRs) fabricated in microstrip technology without ground plane etching
}

\author{
P. Vélez, M. Durán-Sindreu, J. Naqui, J. Bonache and F. Martín \\ CIMITEC-Departament d'Enginyeria Electrònica. Universitat Autònoma de Barcelona. \\ 08193 BELLATERRA (Barcelona). Spain. \\ Phone: 34935813522; Fax: 34935812600. E-mail: Ferran.Martin@uab.es
}

\begin{abstract}
A differential (or balanced) bandpass filter based on open complementary split ring resonators (OCSRRs) coupled through admittance inverters is presented in this paper. Pairs of OCSRRs are symmetrically placed in a mirror configuration between the strips of the differential line and are modeled by means of two series connected parallel resonators. For the differential (odd) mode, there is a virtual ground at the connecting plane between the OCSRR pairs, and the structure is roughly described by the canonical model of a bandpass filter, consisting of a cascade of shunt resonators coupled through admittance inverters. It is demonstrated that, through a proper design of the OCSRR stages, the common mode noise in the vicinity of the differential filter pass band can be efficiently suppressed. Due to the differential mode operation of the filter, it is not necessary to incorporate metallic vias to ground the OCSRRs. Moreover, as compared to other differential filters based on OCSRRs, defected ground structures (DGS) are not present in the proposed filters. To illustrate the potential of the approach, two balanced bandpass filters are designed, fabricated and characterized.
\end{abstract}

KEYWORDS: Split ring resonators, differential bandpass filters, microstrip technology.

\section{CORRESPONDING AUTHOR}

Ferran Martín

Departament d'Enginyeria Electrònica

Universitat Autònoma de Barcelona

08193 BELLATERRA (Barcelona)

Spain

Tel.: 34935813522

Fax.: 34935812600

E-mail: Ferran.Martin@uab.es 


\section{Introduction}

Split ring resonators (SRR) [1] and their dual counterparts (complementary split ring resonators CSRRs) [2,3] have been widely used for the implementation of bulk metamaterials [4,5], transmission line metamaterials [6,7], and many microwave devices and antennas (see [8]), including planar filters [9-19]. The filters reported in [9-19] are based on closed resonators (SRRs or CSRRs) coupled to a host line. However, planar microwave filters can also be implemented by means of the open versions of the above particles, that is, the open split ring resonator (OSRR) [20], and the open complementary split ring resonator (OCSRR) [21]. As compared to SRR/CSRRs, the open resonators (OSRR/OCSRRs) are electrically smaller by a factor of two [20-22]. Moreover, OSRRs and OCSRRs are intrinsically wide band resonators; therefore, these open resonators are of interest for the implementation of moderate and wide band bandpass filters [23-29]. The filters reported in [23-25,29] consist of shunt (OCSRRs) or series (OSRRs) resonators coupled through impedance inverters, either implemented in CPW or microstrip technology, whereas the filters reported in [26-28] are based on a combination of OSRRs and OCSRRs. In the latter filters, the OSRRs and the OCSRRs are described by means of series and parallel resonators, respectively. Despite the fact that some parasitics must be introduced in the model in order to adequately describe the resonators loading a host line, the effects of these parasitics are small, and the filters exhibit the intended (typically Chebyshev) responses to a very good approximation.

In this paper, OCSRRs are used for the implementation of broadband differential bandpass filters. The filter topologies are mirror images of the microstrip single-ended filters reported in [29], consisting of shunt connected OCSRRs coupled through admittance inverters. However, in the proposed balanced filters it is not necessary the use of vias to ground the OCSRRs, since these resonators are effectively grounded for the odd mode due to the presence of the electric wall at the symmetry plane.

This work is justified by the increasing interest in recent years for the development of differential, or balanced, filters [30-37]. The reason is that differential lines and circuits are more robust in front of electromagnetic interference (EMI), and high speed digital circuits are mainly based on balanced lines. Since analog signal processing in such balanced lines and circuits is necessary, and filters are key building blocks for the suppression of interfering signals, spurious suppression, etc., it follows that the design of differential bandpass filters is of fundamental importance.

The paper is organized as follows. In section 2, we will review the OCSRR for completeness, since this resonator is the key building block of the proposed filters. Section 3 is devoted to present the lumped element equivalent circuit model of the differential line loaded with a pair of mirrored OCSRRs, including the models for differential and common mode excitation. The design of the proposed balanced filter, including the simulation and measurement of the filter response for the differential and common mode, is reported in section 4. Finally, the main conclusions are highlighted in section 5.

\section{Topology and circuit model of the OCSRR}

The OCSRR is the open version of the CSRR. The typical topology and circuit model of the OCSRR is depicted in Fig. 1, where the particle terminals (or ports) are indicated in the figure. Between these ports, there is an electric short through the metal between the inner and outer slot rings forming the particle, but there is also a capacitive connection through the capacitances across the slots. Thus, the circuit model of the particle is an open parallel resonant tank, as Fig. 1(b) illustrates. The inductance of the particle, $L_{o}$, is the inductance of the metallic strip between the ring slots, and the capacitance, $C_{c}$, is identical to the capacitance of the CSRR, that is, the capacitance of a disk of radius $r_{o}-c / 2$ (where $r_{o}=r_{e x t}-c-d / 2$ is the averaged radius) surrounded by a metallic plane at a distance $c$ of its edge [7]. Since the inductance of the CSRR is $L_{o}$ /4 [7], it is expected that the resonance frequency of the OCSRR is one half the resonance frequency of the CSRR. The detailed calculation of $C_{c}$ is reported in [7], whereas $L_{o}$ 
is the inductance of the circular CPW structure that results from the OCSRR topology. Notice that the bandwidth of a shunt connected parallel LC resonator increases with the $\mathrm{L} / \mathrm{C}$ ratio. Since the inductance of the OCSRR is relatively large (at least compared to that of the CSRR), broad band filter responses can potentially be achieved with these resonant particles.

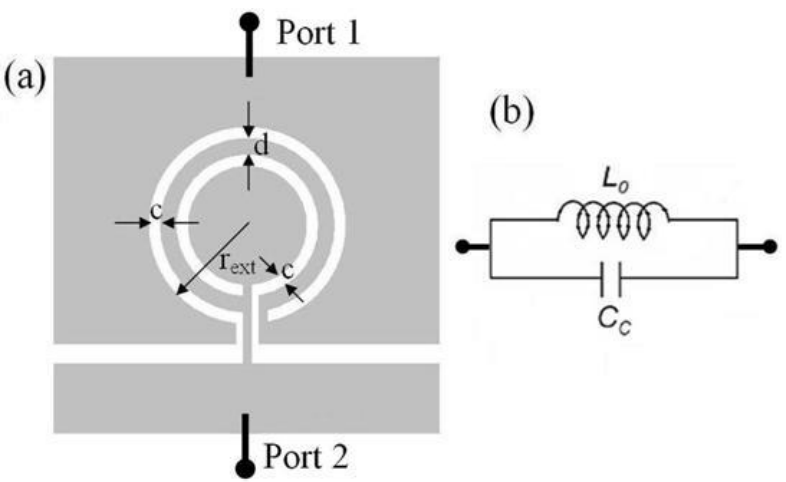

Fig. 1. Typical topology of the OCSRR (a) and equivalent circuit model (b). The two terminals -ports- of the open resonator are indicated.

The previous model can be improved by considering the inductive effect of the metallic strip connecting the inner region of the particle and the metallic region in contact to port 2 . This introduces an inductance in series with the parallel resonator, as reported in [28], and the effect of this inductance/strip is the presence of a short between ports 1 and 2 at a finite frequency (i.e., above the resonance frequency of the OCSRRs). In the bandpass filters based on OSRRs and OCSRRs reported by the authors [26-28], where the OCSRR is shunt connected to the host line (microstrip or CPW), this short produces a transmission zero above the filter pass band. This improves the out of band filter performance (stop band rejection). However, in a first order approximation, the circuit of Fig. 1(b) can be used for design purposes.

\section{Differential microstrip lines loaded with shunt connected OCSRRs}

It was shown in [26] that an OCSRR pair loading a CPW in a symmetric configuration (this is necessary to avoid the slot mode) cannot be merely modeled by a shunt resonator. Some phase shift appears between the input and output ports at OCSRR resonance, and hence phase shifting lines must be introduced for an accurate modeling of the host CPW loaded with the OCSRR pair (in practice such cascaded phase shifting lines have been modeled by means of a series inductance and shunt capacitance). The result is thus a T-circuit model with a shunt parallel resonator and a series inductance, which can be considered a parasitic of the model, as reported in [26]. However, if a microstrip line is loaded with a shunt OCSRR (in this case vias are necessary to effectively ground the particle), the phase shift can be neglected, and the OCSRR-loaded microstrip line can be simply modeled as a parallel LC resonator [26] (including the series inductance to account for the broadband frequency response).

We do also expect similar behavior for a differential microstrip line loaded with a pair of mirrored OCSRRs (Fig. 2a). Notice that for the differential (odd) mode, there is an electric wall at the symmetry plane and hence a virtual ground at that plane. This means that the OCSRRs do not need vias for shunt connection in the differential mode. The complete circuit model describing an OCSRR-loaded differential microstrip line, like the one shown in Fig. 2(a), is shown in Fig. 2(b) [37]. $L_{p}$ and $C_{p}$ model the OCSRR, $L$ accounts for the inductive strip present between the microstrip lines and the center part of the OCSRRs, $C_{l}$ is the capacitance between the inner central strip of the OCSRR and the ground plane; finally, $C_{2}$ is the patch capacitance corresponding to the metallic region surrounding the OCSRRs. The models for the differential and common modes are depicted in Figs. 3(a) and 3(b), respectively. Notice 
that the capacitance $C_{2}$ is grounded for the differential mode and does not play any role. Thus, for this mode, the shunt OCSRR is described by a parallel resonator in series with an inductor, useful to introduce a transmission zero above the differential filter pass band, as mentioned before. Such transmission zero is given by:

$$
f_{Z}^{d d}=\frac{1}{2 \pi} \sqrt{\frac{1}{C}\left(\frac{1}{L}+\frac{1}{L_{p}}\right)}
$$

However, for the common mode, the symmetry plane is an open-circuit, and the effect of the capacitance $C_{2}$ is the presence of two transmission zeroes in the common mode frequency response. Such transmission zeros appear at those frequencies where the shunt reactance, given by

$$
Z_{p}^{C C}(\omega)=j \frac{\omega^{4}\left(C_{p} L_{p} C_{2} L+L L_{p} C_{1} C_{2}+L L_{p} C_{p} C_{1}\right)-\omega^{2}\left(L_{p} C_{2}+L_{p} C_{p}+L C_{2}\right)+1}{\omega\left(\omega^{2}\left(C_{p} L_{p} C_{2}+L_{p} C_{1} C_{2}+L_{p} C_{p} C_{1}\right)-C_{1}-C_{2}\right)}
$$

is null. The first one is useful in order to suppress the common mode noise in the differential filter pass band.

(a)
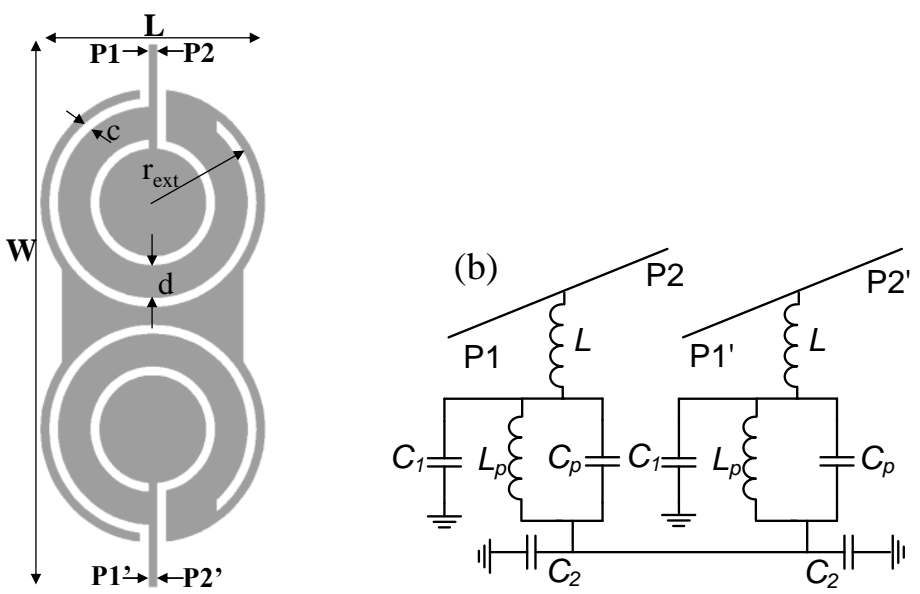

Fig. 2. Typical topology of a differential microstrip line section loaded with a pair of mirrored OCSRRs (a) and lumped element equivalent circuit model (b).

(a)

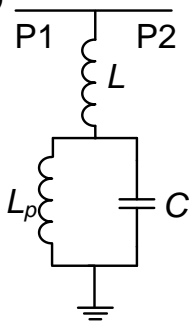

(b)

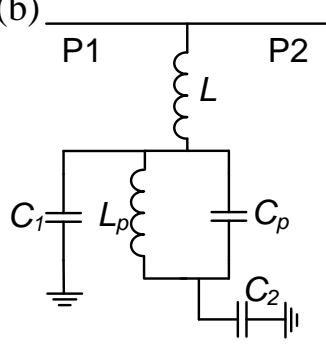

Fig. 3. Circuit model of the structure of Fig. 2(a) for differential (a) and common (b) mode excitations.

Fig. 4(a) depicts the full wave electromagnetic simulation (the Agilent Momentum commercial software has been used) of the reflection coefficient (odd mode) for the topology of Fig. 2(a), where it can be appreciated that the trace of $S_{11}$ roughly lies in the unit conductance circle. This means that the structure can be modeled by a parallel resonator in series with an inductor for the odd mode, as expected. Fig. 4(b) shows the magnitude of the reflection and transmission coefficients for the odd mode (inferred 
from electromagnetic simulation), which is compared with the reflection and transmission coefficients obtained from circuit simulation. The element values of the circuit model $\left(L, L_{p}\right.$, and $\left.C=C_{l}+C_{p}\right)$ are derived from the susceptance slope at $f_{o}$, the frequency of maximum transmission (or the central frequency for the balanced filter), and from the differential mode transmission zero. The good agreement is also appreciable, thus confirming the validity of the differential mode model. Finally, the common mode frequency response is depicted in Fig. 4(c), where again good agreement between circuit and electromagnetic simulation up to $3.5 \mathrm{GHz}$ can be appreciated. The position of the first transmission zero for the common mode can be controlled through the size of the metallic region surrounding the OCSRRs, which effectively determines the value of $C_{2}$.
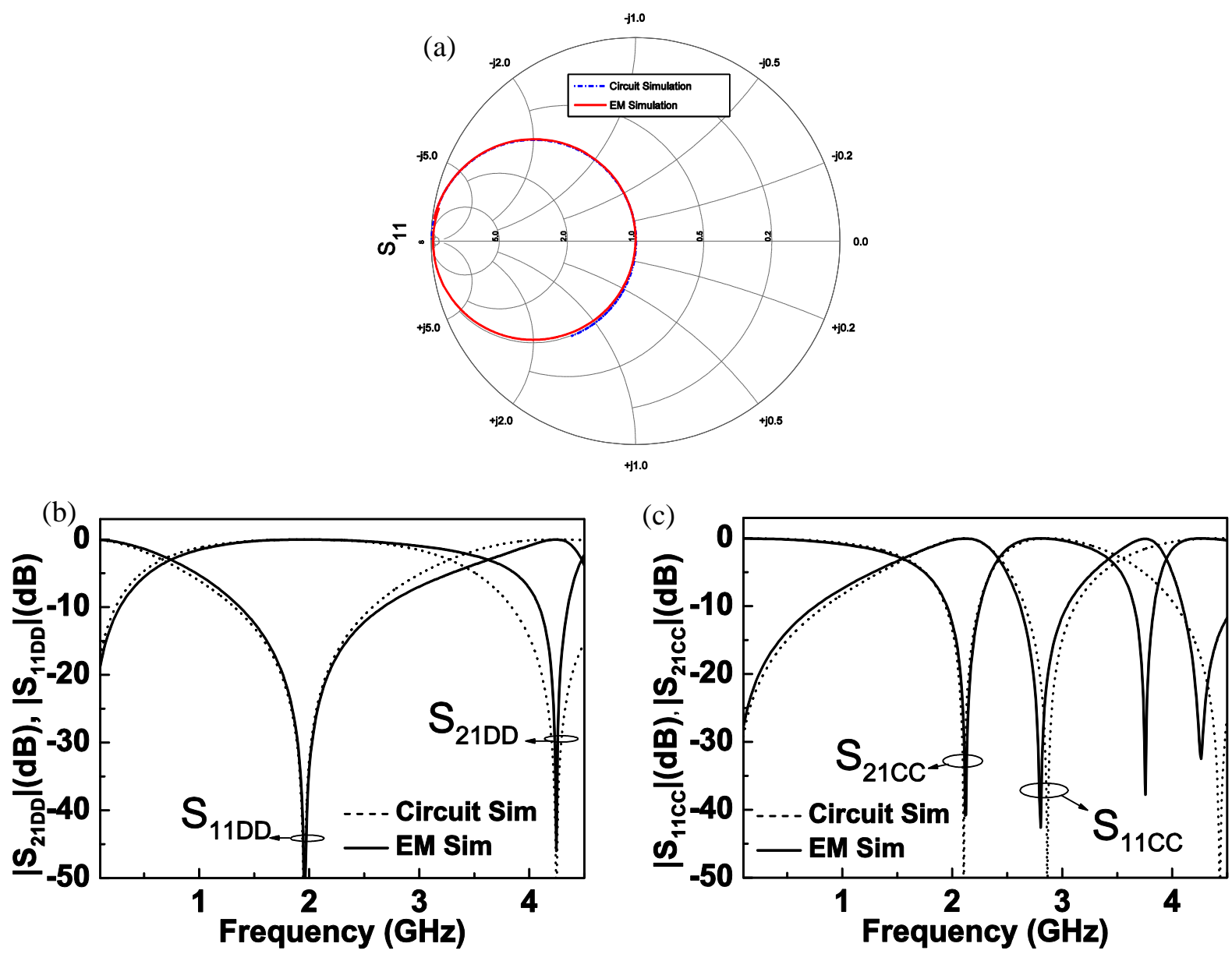

Fig. 4. Frequency response of the structure of Fig. 2. (a) Electromagnetic and circuit simulation of the reflection coefficient depicted in the Smith Chart; (b) magnitude of the differential mode insertion and return loss and (c) magnitude of the common mode insertion and return loss. Dimensions (in reference to Fig. $2 \mathrm{a}$ ) are: $r_{\text {ext }}=1.9 \mathrm{~mm}, c=0.16 \mathrm{~mm}$ and $d=0.6 \mathrm{~mm}$. The considered substrate is Rogers RO3010 with thickness $h=0.635 \mathrm{~mm}$ and dielectric constant $\varepsilon_{r}=10.2$. The circuit element values are: $L=1.15 \mathrm{nH}, L_{p}=4.239 \mathrm{nH}, C_{p}=0.2 \mathrm{pF}, C_{l}=1.349 \mathrm{pF}, C_{2}=0.85 \mathrm{pF}$.

\section{Differential filters based on OCSRRs and results}

\subsection{Example 1}

The first proposed differential filter is an order-3 Chebyshev band pass filter with central frequency $f_{o}=2$ $\mathrm{GHz}, 0.03 \mathrm{~dB}$ ripple, and fractional bandwidth of $60 \%$, including a transmission zero at $f_{z}^{d d}=4 \mathrm{GHz}$. This filter is implemented by means of shunt resonators coupled through admittance inverters (see Fig. 5), following the theory reported in [38]. For these specifications, considering identical resonators with 
a value of inductance and capacitance of $L_{p i}=4.39 \mathrm{nH}$ and $C_{p i}=1.44 \mathrm{pF}$, respectively, the characteristic admittance of the inverters is found to be $J_{01}=J_{34}=0.0166 \mathrm{~S}$, and $J_{12}=J_{23}=0.0118 \mathrm{~S}$.

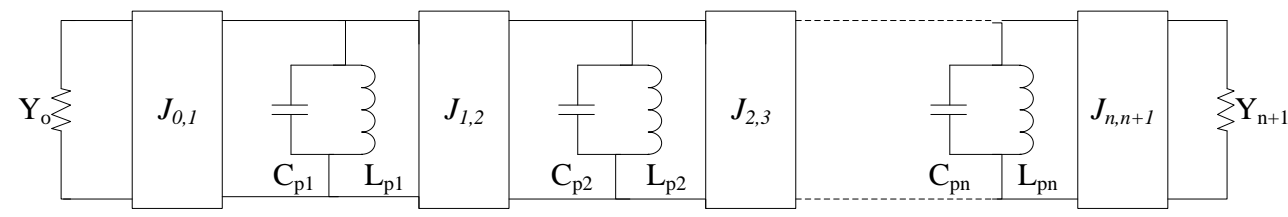

Fig. 5. Order-n bandpass filter network with admittance inverters and shunt LC resonators.

The proposed differential filter alternates the differential OCSRR-loaded line sections with $\lambda / 4$ lines corresponding to the admittance inverters ( $\lambda$ is the wavelength at the central filter frequency). The filter topology is depicted in Fig. 6. As compared to previous OCSRR and OSRR based differential filters [37], the proposed filter does not include defect ground structures (DGS) in the ground plane. This represents a significant progress in applications where the device must rest on top of a metallic holder, whereas the area is not significantly increased by the use of the $\lambda / 4$ lines. The design process of the filter is similar to that reported in [37]. For the OCSRRs, the elements of the model of Fig. 3(a) $\left(L, L_{p}\right.$, and $C$ $=C_{1}+C_{p}$ ) are derived from the susceptance slope at $f_{o}$ (i.e., by forcing it to be equal to that of the LC tank giving the ideal Chebyshev response), and from the differential mode transmission zero (which is another filter specification). Once $L, L_{p}$, and $C$ are known, the OCSRRs are synthesized with the help of the model reported in [21] and a parameter extraction procedure similar to that reported in [28]. From this model, the OCSRR capacitance, $C_{p}$, can be estimated and hence $C_{l}$ can be derived. Finally, $C_{2}$ is adjusted to the required value to force the common mode transmission zero at $f_{o}$. The metallic region surrounding the OCSRRs is then expanded or contracted to adjust the common mode transmission zero to that value (the initial size is inferred from the parallel plate capacitor formula). Following this procedure, the element values and the layout of the shunt branch (OCSRR) have been inferred. Since the distance between the strips of the differential line is large, the coupling between them is negligible and the strip widths are calculated from the characteristic impedance of the inverters as if the lines were isolated. The photograph of the designed filter, fabricated following a standard photo/mask etching technique, is also depicted in Fig. 6.

(a)

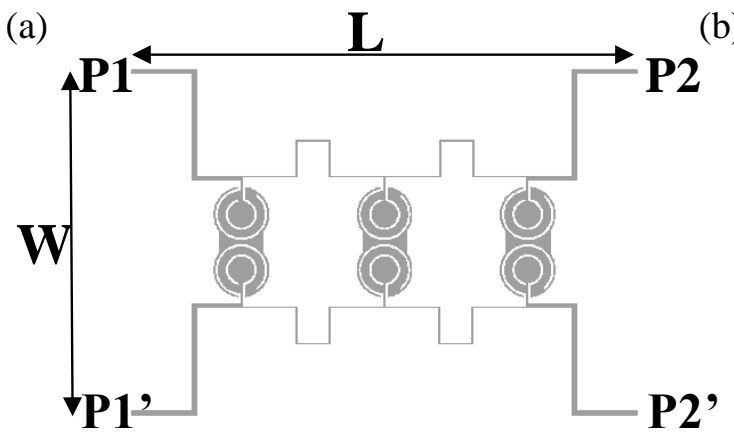

(b)

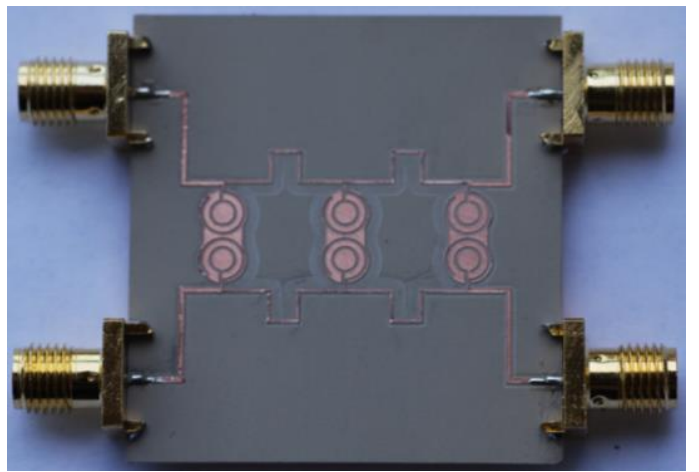

Fig. 6. Topology (a) and photograph (b) of the proposed differential bandpass filter of example 1. The substrate is the Rogers $R O 3010$ with thickness $h=0.635 \mathrm{~mm}$ and dielectric constant $\varepsilon_{l}=10.2$. For the OCSRRs, $r_{e x t}=1.9 \mathrm{~mm}, c=0.16 \mathrm{~mm}$ and $d=0.6$ $\mathrm{mm}$. The width of the transmission line inverters is $0.359 \mathrm{~mm}$ for the external ones and $0.161 \mathrm{~mm}$ for the internal ones. The total area is $9.56 \mathrm{~cm}^{2}(L=37.2 \mathrm{~mm}$ and $\mathrm{W}=25.7 \mathrm{~mm})$.

The simulated frequency response of the proposed filter, inferred from the Agilent Momentum full wave electromagnetic solver, considering differential ports, is depicted in Fig. 7, and it is compared to the ideal Chebyshev response. Since ideal inverters are not being considered, the simulated response exhibits same discrepancy, as revealed by the smaller bandwidth. Moreover, this effect is enhanced by the transmission zero, which improves the filter stopband performance. Nevertheless, bandwidth narrowing can be compensated if necessary by over-dimensioning the nominal bandwidth. The 
agreement with the measured response (obtained by means of the 4-port Agilent PNA N5221A vector network analyzer) and with the circuit simulation (differential and common modes) is also good. The differential filter performance is good, with a measured in-band insertion loss smaller than $0.6 \mathrm{~dB}$ and return loss better than $18 \mathrm{~dB}$ in the pass band. Fig. 7 includes also the simulated and measured common mode response, where it can be appreciated that a strong rejection of the common mode $(35 \mathrm{~dB})$ in the differential filter pass band edges is obtained.
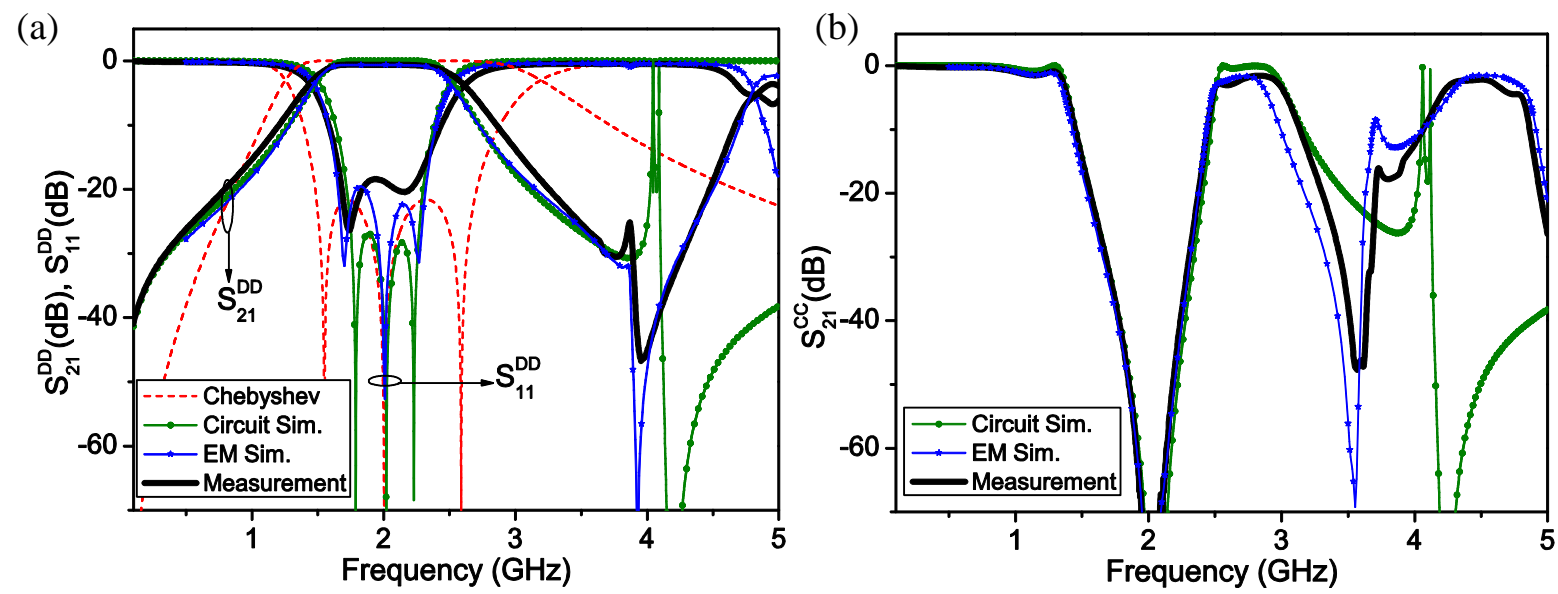

Fig. 7. Differential-mode insertion and return loss (a), and common-mode insertion loss (b) of the designed order-3 balanced bandpass filter based on OCSRR and admittances inverters.

\subsection{Example 2}

To overcome the previous problem relative to the limited bandwidth of the inverters, causing a lower fractional bandwidth than the nominal one, we have considered another design approach, reported in [8]. The idea consists in considering the elemental cell as composed of the shunt resonator sandwiched between a pair of matched $\lambda / 8$ lines. If the $-3 \mathrm{~dB}$ frequencies of each cell are designated as $\omega_{1}$ and $\omega_{2}$, it follows that the impedance of the shunt branch (resonator) is $+Z_{o} / 2$ and $-Z_{o} / 2$ at $\omega_{1}$ and $\omega_{2}$, respectively, and infinity at $\omega_{0}$. Hence, once $\omega_{1}$ and $\omega_{2}$ are known, the element values can be inferred. To determine $\omega_{1}$ and $\omega_{2}$, the following expression is used [8]:

$$
\Delta_{i}=\frac{2 F B W}{g_{i}}
$$

where $F B W$ is the filter fractional bandwidth, $g_{i}$ 's are the elements of the low pass filter prototype, and $\Delta_{\mathrm{i}}$ is the $-3 \mathrm{~dB}$ bandwidth of each filter cell.

In this second example, the intended response is an order-3 Chebyshev, with $F B W=35 \%$ and ripple $0.007 \mathrm{~dB}$. The element values of the normalized low pass filter prototype are: $g_{1}=g_{3}=0.5869$ and $g_{2}=$ 0.9328. The resulting element values of the resonators for cells 1 and 3 are: $L_{p}=2.065 \mathrm{nH}, C_{p}=3.065$ $\mathrm{pF}$ and $L=0.5427 \mathrm{nH}$. The rest of the design process is identical to the one of example 1. Figure 8 shows the topology and photograph of the designed filter, and the frequency response of the filter is depicted in Fig. 9. The agreement between circuit, electromagnetic simulation and measurement is very good, and common mode noise rejection in the differential filter pass band is significant (more than 25 $\mathrm{dB})$. In this case, the differential filter bandwidth agrees with the nominal value to a good approximation. Nevertheless, the frequency selectivity of the designed filter at the upper band edge is better than the Chebyshev response due to the presence of the transmission zeros above the differential filter pass band. 
(a)

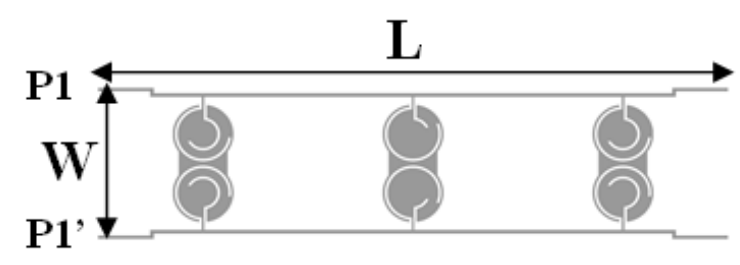

(b)

$\mathbf{P 2}$

P2'

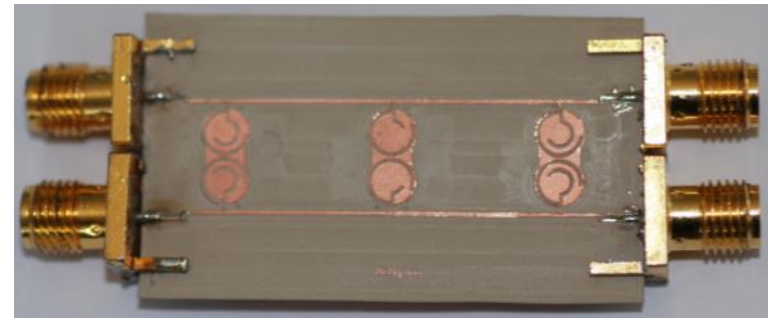

Fig. 8. Topology (a) and photograph (b) of the proposed differential bandpass filter of example 2. The substrate is the Rogers $R O 3010$ with thickness $h=0.254 \mathrm{~mm}$ and dielectric constant $\varepsilon_{r}=10.2$. For the OCSRRs, $r_{e x t}=2.06 \mathrm{~mm}, c=0.16 \mathrm{~mm}$ and $d=0.6$ $\mathrm{mm}$. The total area is $4.52 \mathrm{~cm}^{2}(L=42.7 \mathrm{~mm}$ and $\mathrm{W}=10.6 \mathrm{~mm})$.
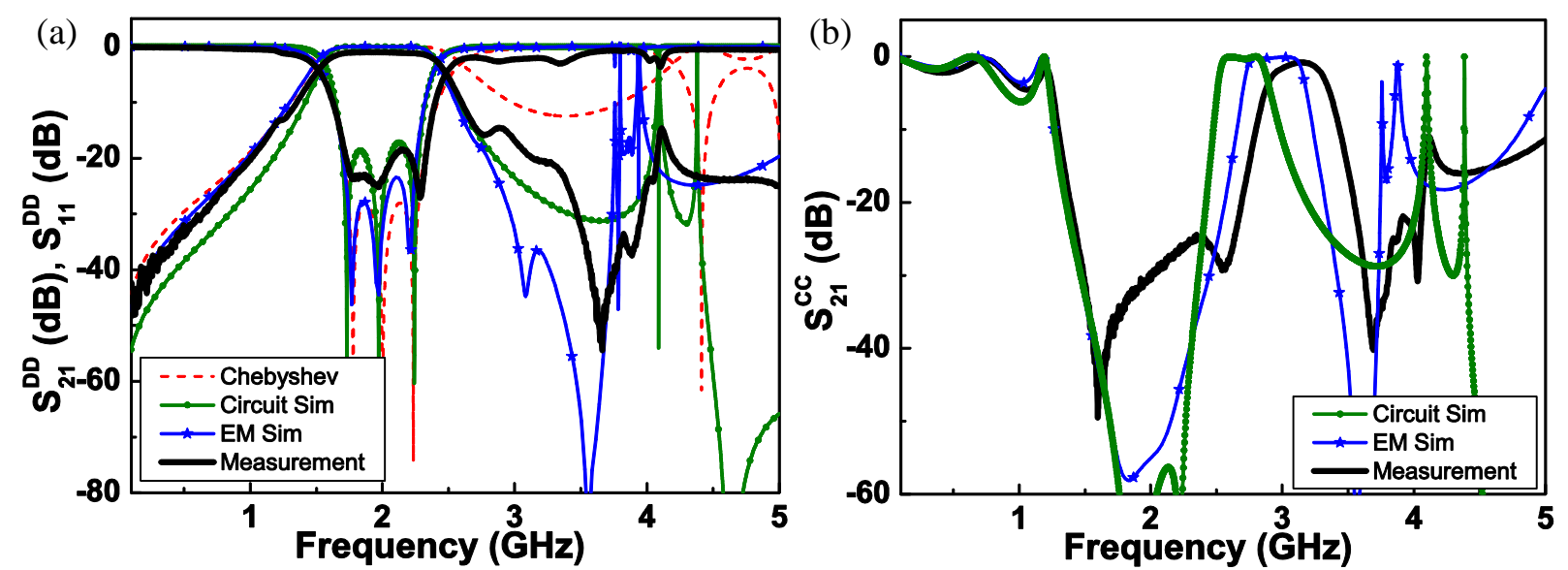

Fig. 9. Differential-mode insertion and return loss (a), and common-mode insertion loss (b) of the designed order-3 balanced bandpass filter of example 2 based on OCSRR cascaded between matched $\lambda / 8$ lines.

\section{Conclusions}

We have proposed a strategy for the design of common-mode suppressed wideband balanced bandpass filters using OCSRRs. Device design is based on the reported equivalent circuit model. This model is useful to adjust the transmission zero for the differential mode response, of interest to improve the filter selectivity above the pass band of interest. Also, the model is used to control the common mode transmission zero, which must be located at the center of the differential filter pass band in order to achieve efficient common mode suppression. Filter size, easy design and fabrication (vias are not required and the ground plane is kept unaltered), and efficient common-mode suppression are relevant advantages of this approach.

\section{Acknowledgements}

This work has been supported by MINECO-Spain (projects TEC2010-17512, CSD2008-00066 and TEC2011-13615-E) and by AGAUR-Generalitat de Catalunya, through project 2009SGR-421. Paris Vélez and Jordi Naqui are in debt to MINECO (Spain) for supporting their work through the FPU grants AP2010-0467 and AP2010-0431, respectively.

\section{References}

1. J.B. Pendry, A.J. Holden, D.J. Robbins and W.J. Stewart, "Magnetism from conductors and enhanced nonlinear phenomena", IEEE Transactions Microwave Theory Tech., vol. 47, pp. 2075-2084, Nov.1999. 
2. F. Falcone, T. Lopetegi, J.D. Baena, R. Marqués, F. Martín, and M. Sorolla, "Effective negative-epsilon stopband microstrip lines based on complementary split ring resonators", IEEE Microwave and Wireless Components Letters, vol. 14, pp. 280-282, June 2004.

3. F. Falcone, T. Lopetegi, M.A.G. Laso, J.D. Baena, J. Bonache, R. Marqués, F. Martín, M. Sorolla, "Babinet principle applied to the design of metasurfaces and metamaterials", Phys. Rev. Lett., vol. 93, paper 197401, November 2004.

4. D.R. Smith, W.J. Padilla, D.C. Vier, S.C. Nemat-Nasser and S. Schultz, "Composite medium with simultaneously negative permeability and permittivity", Phys. Rev. Lett., vol. 84, pp. 4184-4187 (2000).

5. R.A. Shelby, D.R. Smith and S. Schultz, "Experimental verification of a negative index of refraction", Science, vol. 292, pp. 77-79 (2001).

6. F. Martín, F. Falcone, J. Bonache, R. Marqués and M. Sorolla, "Split ring resonator based left handed coplanar waveguide", Appl. Phys. Lett., vol. 83, pp. 4652-4654, December 2003.

7. J.D. Baena, J. Bonache, F. Martín, R. Marqués, F. Falcone, T. Lopetegi, M.A.G. Laso, J. García, I Gil, M. Flores-Portillo and M. Sorolla, "Equivalent circuit models for split ring resonators and complementary split rings resonators coupled to planar transmission lines", IEEE Transactions on Microwave Theory and Techniques, vol. 53, pp. 1451-1461, April 2005.

8. R. Marqués, F. Martín and M. Sorolla, Metamaterials with Negative Parameters: Theory, Design and Microwave Applications, John Wiley, 2008.

9. F. Martín, F. Falcone, J. Bonache, T. Lopetegi, R. Marqués and M. Sorolla, "Miniaturized CPW stop band filters based on multiple tuned split ring resonators" IEEE Microwave and Wireless Components Letters, vol. 13, pp. 511-513, December 2003

10. J. García-García, F. Martín, F. Falcone, J. Bonache, I. Gil, T. Lopetegi, M.A.G. Laso, M. Sorolla, R. Marqués, "Spurious passband suppression in microstrip coupled line band pass filters by means of split ring resonators", IEEE Microwave and Wireless Components Letters, vol. 14, pp. 416-418, September 2004.

11. J. Bonache, F. Martín, J. García-García, I. Gil, R. Marqués and M. Sorolla, "Ultra wide band pass filtres (UWBPF) based on complementary split rings resonators", Microwave and Optical Technology Letters, vol. 46, pp.283-286, August 2005.

12. J. Bonache, I. Gil, J. García-García, F. Martín, "Novel Microstrip Band Pass Filters Based on Complementary Split Rings Resonators", IEEE Transactions on Microwave Theory and Techniques, vol. 54, pp. 265-271, January 2006.

13. J. García-García, J. Bonache, I. Gil, F. Martín, M.C. Velazquez-Ahumada and J. Martel, "Miniaturized microstrip and CPW filters using coupled metamaterial resonators", IEEE Transactions on Microwave Theory and Techniques, vol. 54, pp. 2628-2635, June 2006.

14. P. Mondal, M.K. Mandal, A. Chaktabarty, S. Sanyal, "Compact bandpass filters with wide controllable fractional bandwidth", IEEE Microwave and Wireless Components Letters, vol. 16 pp. 540-542, 2006.

15. M. Gil, J. Bonache, J. García-García, J. Martel and F. Martín, "Composite Right/Left Handed (CRLH) Metamaterial Transmission Lines Based on Complementary Split Rings Resonators (CSRRs) and Their Applications to Very Wide Band and Compact Filter Design”, IEEE Transactions on Microwave Theory and Techniques, vol. 55, pp. 1296-1304, June 2007.

16. I. Gil, F. Martín, X. Rottenberg and W. De Raedt, "Tunable stop-band filter at Q-band based on RF-MEMS metamaterials", Electronics Letters, vol. 43, p. 1153, October 2007.

17. M. Gil, J. Bonache, F. Martín, "Metamaterial filters: a review", Metamaterials, vol. 2, pp. 186-197 December 2008.

18. A.L. Borja, J. Carbonell, V.E. Boria, J. Cascón, D. Lippens, "Synthesis of compact and highly selective filters via metamaterial-inspired coplanar waveguide line technologies", IET Microwaves Ant. Propag., vol. 4, pp. 1098-1104, Aug. 2010.

19. A. L. Borja, J. Carbonell, V. E. Boria, J. Cascon, D. Lippens, "A 2\% Bandwidth C-Band Filter Using Cascaded Split Ring Resonators”, IEEE Antennas Wireless Prop. Lett., vol. 9, pp. 256-259, 2010.

20. J. Martel, R. Marqués, F. Falcone, J.D. Baena, F. Medina, F. Martín and M. Sorolla, "A new LC series element for compact band pass filter design", IEEE Microwave Wireless Components Letters, vol. 14, pp. 210-212, May 2004

21. A. Velez, F. Aznar, J. Bonache, M.C. Velázquez-Ahumada, J. Martel and F. Martín, “Open complementary split ring resonators (OCSRRs) and their Application to Wideband CPW Band Pass Filters", IEEE Microwave and Wireless Components Letters, vol. 19, pp. 197-199, April 2009.

22. F. Aznar, A. Vélez, M. Durán-Sindreu, J. Bonache and F. Martín “Open complementary split ring resonators (OCSRRs): physics, modelling and analysis", Microwave and Optical Technology Letters, vol. 52 (7), pp. 1520-1526, July 2010.

23. J. Martel, J. Bonache, R. Marqués, F. Martín and F. Medina, "Design of Wide-band Semi-lumped Bandpass Filters Using Open Split Ring Resonators", IEEE Microwave and Wireless Components Letters, vol. 17, pp. 28-30, Jan. 2007. 
24. A. Vélez, F. Aznar, M. Durán-Sindreu, J. Bonache and F. Martín, "Stop-Band and Band-Pass Filters in Coplanar Waveguide Technology Implemented by Means of Electrically Small Metamaterial-Inspired Open Resonators", IET Microwaves, Antennas and Propagation, vol. 4, pp. 712-716, Jun. 2010.

25. A. Vélez, F. Aznar, M. Durán-Sindreu, J. Bonache and F. Martín,’Tunable Coplanar Waveguide (CPW) Band-stop and Band-pass Filters based on Open Split Ring Resonators and Open Complementary Split Ring Resonators", IET Microwaves Antennas and Propagation, vol. 5, pp. 277-281, Feb. 2011.

26. M. Durán-Sindreu, A. Vélez, F. Aznar, G. Sisó, J. Bonache and F. Martín, “Application of Open Split Ring Resonators and Open Complementary Split Ring Resonators to the Synthesis of Artificial Transmission Lines and Microwave Passive Components", IEEE Trans. Microwave Theory and Techniques, vol. 57, pp. 3395-3403, Dec. 2009.

27. M. Durán-Sindreu, A. Vélez, G. Sisó, J. Selga, P. Vélez, J. Bonache, and F. Martín "Recent advances in metamaterial transmission lines based on split rings", Proceedings of the IEEE, vol. 99, pp. 1701-1710, October 2011.

28. M. Durán-Sindreu, P. Vélez, J. Bonache and F. Martín, "Broadband Microwave Filters Based on Open Split Ring Resonators (OSRRs) and Open Complementary Split Ring Resonators (OCSRRs): improved models and design optimization", Radioengineering, vol. 20, pp. 775-783, Dec. 2011.

29. P. Vélez, J. Naqui, M. Durán-Sindreu, J. Bonache, and F. Martín, "Broadband microstrip bandpass filter based on open complementary split ring resonators (OCSRRs)", Int. Journal of Antennas and Propagation, Volume 2012, Article ID 174023, 6 pages, doi:10.1155/2012/174023, 2012.

30. C. H. Wu, C. H. Wang, and C. H. Chen, "Novel balanced coupled-line bandpass filters with common-mode noise suppression," IEEE Trans. Microw. Theory Techn., vol. 55, pp. 287-295, Feb. 2007.

31. B. L. Teck, and Z. Lei, "A differential-mode wideband bandpass filter on microstrip line for UWB applications," IEEE Microw. Wireless Compon. Lett., vol. 19, pp. 632-634, Oct. 2009.

32. J. Shi, and Q. Xue, "Novel balanced dual-band bandpass filter using coupled stepped-impedance resonators," IEEE Microw. Wireless Compon. Lett., vol. 20, pp. 19-21, Jan. 2010

33. J. Shi, and Q. Xue, "Dual-band and wide-stopband single-band balanced bandpass filters with high selectivity and common-mode suppression," IEEE Trans. Microw. Theory Techn., vol. 58, pp. 2204-2212, Aug. 2010.

34. J. Shin, and Q. Xue, "Balanced bandpass filters using center-loaded half-wavelength resonators," IEEE Trans. Microw. Theory Tech., vol. 58, no. 4, pp. 970-977, Apr. 2010.

35. C.-H. Wu, C.-H. Wang, and C. H. Chen, "Stopband-extended balanced bandpass filter using coupled stepped-impedance resonators," IEEE Microw. Wireless Compon. Lett., vol 17, no. 7, pp. 507-509, Jul. 2007.

36. C.-H. Lee, C.-I. G. Hsu, and C.-C. Hsu, "Balanced dual-band BPF with stub-loaded SIRs for common-mode suppression," IEEE Microw. Wireless Compon. Lett., vol. 20, no. 2, pp. 70-73, Feb. 2010.

37. P. Vélez, J. Naqui, A. Fernández-Prieto, M. Durán-Sindreu, J. Bonache, J. Martel, F. Medina, and F. Martín, "Differential Bandpass Filter with Common Mode Suppression based on Open Split Ring Resonators and Open Complementary Split Ring Resonators", IEEE Microwave and Wireless Components Letters, vol. 23, no. 1, pp. 22-24, Jan. 2013.

38. J-S. Hong and M.J. Lancaster, Microstrip filters for RF/microwave applications, John Wiley \& Sons, Inc., New York, 2001. 